\title{
An Empirical Study on the Synergic Relationship between the Regional Sci-Tech Innovation Platform and the Development of Strategic Emerging Industries
}

\author{
Weiran Zhang \\ School of Business Administration, South China University of Technology, Guangzhou, China \\ Email: 13246803321@163.com
}

How to cite this paper: Zhang, W.R. (2018) An Empirical Study on the Synergic Relationship between the Regional Sci-Tech Innovation Platform and the Development of Strategic Emerging Industries. American Journal of Industrial and Business Management, 8, 747-759.

https://doi.org/10.4236/ajibm.2018.83051

Received: February 7, 2018

Accepted: March 26, 2018

Published: March 29, 2018

Copyright $\odot 2018$ by author and Scientific Research Publishing Inc. This work is licensed under the Creative Commons Attribution International License (CC BY 4.0).

http://creativecommons.org/licenses/by/4.0/

\begin{abstract}
The construction of regional STI platform is a significant carrier for cultivating and developing strategic emerging industries, and the synergistic coupling between the layout of such construction and industrial development's stage and demands is the prerequisite and basis for realizing industry support of STI platform. From both the width and intensity of the synergistic coupling, this paper constructs the synergetic relationship measure model, and verifies its effectiveness and feasibility through the example of Guangdong Province. The survey finds that in the synergetic relationship between them in Guangdong, there are large regional differences, embodied in four types: platformdriven, industrial spillover, synchronous development and benign interaction, and the width and intensity of the synergistic coupling is relatively low while a benign interaction coupling mechanism has not yet been formed. This study provides a scientific basis for effectively measuring the degree of the synergistic coupling between regional STI platform and industries, and optimizing the layout of STI platform construction.
\end{abstract}

\section{Keywords}

Sci-Tech Innovation Platform, Strategic Emerging Industries, Synergistic Coupling

\section{Introduction and Literature Review}

Strategic emerging industry is a high-tech industry based on the breakthrough of core technology in China. It is of great significance to cultivate and develop 
strategic emerging industry to realize the upgrading of industrial structure. The fundamental way to accelerate the development of emerging industries is to break through the core technology and improve the innovation ability of enterprises. However, this has been restricted by the low efficiency of the allocation of scientific and technological resources [1]. For this, regional STI platform arises at the historic moment, its core purpose is to effectively organize the innovation elements to gather in the enterprise, build and perfect the support service system that promotes enterprise technical innovation, vigorously promote the combination of Industry-University-Research institutions, enhance our capacity for independent innovation, it is the key enterprises around the industry core technology breakthrough and other major target the carrier of long-term strategic cooperation [2] [3]. STI platform and strategic emerging industries are as two national economic and social important subsystems, closely the endogenous interactive relationship exists between two, and through their respective coupling elements and promote each other, coordinate with each other, forming a kind of interactive coupling relationship, the two coupled coordination development is conducive to regional sustainable rapid growth of knowledge economy in China.

The article is organized as follows: The first paragraph is "Introduction and Literature review"; The second paragraph is "The cooperative relationship between Regional STI platform and strategic emerging industry and its measurement method"; The third paragraph is "Empirical research and results discussion"; The fourth paragraph is "Conclusions and recommendations".

The research on regional STI platform and strategic emerging industries has been gradually heated up in recent years. Before this, scholars at home and abroad mainly focused on the relationship between technological innovation and industrial development, Foreign scholar Nelson (1997) pointed out that the fundamental driving force of industry is innovation [4]; Yue Zhonggang (2014) believes that the emerging technology chain is the core of industrial development, and the coordinated development of the two is the key to the growth of strategic emerging industries [5]. Huang Lucheng (2014) analyzed the co-evolution law of emerging technologies and emerging industries with the analysis paradigm of technological innovation economics and co-evolution theory [6]. There are also some scholars who have incorporated the element of STI platform into the research framework of technological innovation and industry. Wang Xiuting (2007) argued that regional STI platform is an urgent need to promote industrial technological capability to realize the independent development of industry. It is the basis of the production and transfer of the achievements of Industrial Science and technology innovation, and it is an important carrier to conquer the common technical problems of the industry and enhance the technical ability of the industry [7]. Zheng Xiaobi et al. (2011) demonstrated the main significance and feasibility of regional STI platform to promote industrial transformation and upgrading [8]. Wang Shaoyong (2014) pointed out that the government's investment in innovation and regional STI platform construction 
can improve the innovation efficiency of strategic emerging industries, create an industrial innovation driven development mode, and provide a continuous core technology for the development of strategic emerging industries [9]. In general, the study on regional STI platform and strategic emerging industries made a positive contribution to the related research, but still the following deficiencies: First, the above research focuses on the qualitative research on the relationship between the two, but it lacks the research of synergistic effect; Secondly, there are few studies on the evaluation of the synergistic effect of the two, and the evaluation methods are not yet sound. However, between different areas of China resources vary, it will lead to regional STI platform and strategic emerging industry collaborative coupling development is not balanced. It is of great theoretical and practical significance to have a comprehensive understanding of the status of the coordinated development of China's regional STI platform and industry, and to formulate relevant policies for industrial development or technology development.

In view of the lack of theoretical research on regional STI platform and the importance of co-coupling development of regional STI platform and strategic emerging industries, in this paper, the coupling theory of physics is used to construct the evaluation model of the synergistic coupling degree between the two. Taking Guangdong as an example, this paper measures the degree of synergistic development between the two and can effectively measure the status of the coordinated development of China's regional STI platform and industry and provides a scientific basis for optimizing the layout of the innovation platform.

\section{The Cooperative Relationship between Regional STI Platform and Strategic Emerging Industry and Its Measurement Method}

\subsection{Collaborative Matching Process and Internal Mechanism}

Coupling refers to two or more than two systems or modes of motion, through mutual coordination, mutual promotion and other benign interaction to produce dynamic synergistic amplification effect, and promote the whole system from disorder to orderly process [10]. Strategic emerging industries and regional STI platform are important subsystems of regional knowledge economy. Both of them promote each other and grow together. On the one hand, the innovation platform will form new production technologies, optimize the allocation of resources, promote the technological transformation of traditional industries, promote the upgrading of industrial structure, and promote the development of strategic emerging industries; On the other hand, because the stress effects of technological progress, market demand and government behavior, strategic emerging industry must change the mode of growth, through continuous technological innovation, industrial agglomeration, and promote regional economic transformation and regional transformation and promoting the further development of technology and regional STI platform and the introduction of talent, 
thus forming a virtuous coordinated development of both the cycle. The innovation platform and the strategic emerging industries are realizing the continuous coupling through technological innovation [11].

The synergistic development of strategic emerging industries and regional STI platform is reflected in the continuous enhancement of the coupling width and intensity of the two coupling. Coupling width refers to the interaction coupling between two subsystems, and the amplitude and width of interface affected by system interaction, which is embodied in synchronization and consistency between systems. The coupling strength refers to the two systems in the coupling interface interaction and coordination of depth and level. Usually, the relationship between strategic emerging industry and innovation platform is more closely related to technology, talent and information. The smaller the difference of development level between them is, the higher the synchronization and consistency of them is. The higher the interface amplitude and breadth level is, so the greater the coupling width is. In the strategic emerging industry and regional STI platform in many ways based on the organic connection, If the two are clearly transferred, infiltrated, and spread in many aspects, such as technology, talent, information, and so on. The greater the radiation and support is between the two, The strategic emerging industries through industrial impact ability to promote regional innovation platform upgrade, The innovation platform plays a supporting role in the development of strategic emerging industries through technology transfer and spillover, This means that the synergy between the two is more significant, Then the depth and level of correlation and coordination on the coupling cross section are higher, the coupling strength is greater [12].

\subsection{Collaborative Relationship Measurement Model Construction}

Based on system theory, we believe that synergy refers to the harmonious symbiotic relationship between subsystems in the development of complex system, and the degree of synergy coupling is an important index [13] to measure the coordinated development between different systems [13]. First, we measure the comprehensive development level of them. Then we build a synergistic coupling width and synergistic coupling strength model of regional STI platform and strategic emerging industries, so as to evaluate the coordinated development level of them.

\subsubsection{Regional STI Platform and Strategic Emerging Industries Development Level Measure Model}

Suppose the variable $U_{i}(i=1,2, \ldots N)$ is the comprehensive order parameter of the I subsystem of the platform and the industry coupling system, and $U_{i j}$ is the $j$ index of the order parameter of the I. The value of $U_{i j}$ represents the contribution to the efficiency of the system, reflecting the satisfaction of a single indicator to the target value. Because the platform and the industry are two different and interacting subsystems, the total contribution to the order degree of each order parameter in the subsystem can be realized through the integration methodology. 
This paper uses the linear weighting method to realize.

$$
U_{i}=\sum_{j=1}^{m} W_{i j} u_{i j}
$$

Formula (1): $U_{i}$ is the contribution of the subsystem to the order of the total system. In this paper, $U_{1}$ is used to express the comprehensive index of the construction of the technological innovation platform, $U_{2}$ represents the comprehensive index of the growth of the strategic emerging industry, $W_{i j}$ represents the weight of each variable, and $\mathrm{m}$ is the number of indicators. And $\sum_{j=1}^{m} w_{i j}=1$.

\subsubsection{Collaborative Coupling width Measurement Model}

Based on the comprehensive evaluation of the development level of strategic emerging industries and regional innovation platform, we can further calculate the synergistic coupling width between the comprehensive evaluation value $U_{1}$ of regional innovation platform and the comprehensive evaluation value $U_{2}$ of strategic emerging industries [14]. If the difference between $U 1$ and $U_{2}$ is smaller, the synergistic coupling width between $U_{1}$ and $U_{2}$ is higher, and the greater the deviation between $U_{1}$ and $U_{2}$, the lower the synergistic coupling width of $U_{1}$ and $U_{2}$.

$$
C V=\frac{S}{1 / 2 \times\left[U_{1}+U_{2}\right]}=\left\{2 \times\left(1-\frac{U_{1} \times U_{2}}{\left(\frac{U_{1}+U_{2}}{2}\right)^{2}}\right)\right\}^{1 / 2}
$$

In formula (2), $C V$ is the deviation coefficient of $U_{1}$ and $U_{2}$; $\mathrm{S}$ is the standard deviation of $U_{1}$ and $U_{2}$. In statistical significance, the synergistic coupling width between $U_{1}$ and $U_{2}$ is in inverse proportion to the difference between $U_{1}$ and $U_{2}$ [15], Therefore, $\left(U_{1} \times U_{2}\right) /\left(\frac{U_{1}+U_{2}}{2}\right)^{2}$ can be used to represent the synergistic coupling width of $U_{1}$ and $U_{2}$, and if the CO Coupling width of $U_{1}$ and $U_{2}$ is recorded as $\mathrm{C}$, there are:

$$
C=\frac{U_{1} \times U_{2}}{\left(\frac{U_{1}+U_{2}}{2}\right)^{2}}
$$

Thus, synergistic coupling width of $C$ in $[0,1]$, when $C=1$, regional STI platform and strategic emerging industries as benign resonance coupling; when $C=$ 0 , regional STI platform has nothing to do with the strategic emerging industries.

\subsubsection{Collaborative Coupling Strength Measurement Model}

The synergetic coupling width is only concerned with the synchronization and consistency between the systems. It is difficult to reflect the "overall effectiveness" of the collaborative development of the system [16]. In order to overcome 
the defect of the synergistic coupling width model, based on the existing research, this paper further constructs a synergistic coupling strength model that can reflect the coordinated development of the coupling system:

$$
\begin{gathered}
D=\sqrt{C \times T} \\
T=\alpha U_{1}+\beta U_{2}
\end{gathered}
$$

In the above equation: $D$ is a synergistic coupling strength value; $T$ for the comprehensive coordination index of regional $S T I$ platform and strategic emerging industries, reflecting the overall synergistic effect between the two systems [3]; $\alpha$ and $\beta$ as unknown parameters, reflect the relative importance of the two systems, regional STI platform and strategic emerging industries is equally important, so $\alpha=\beta=0.5$.

\section{Empirical Research and Results Discussion}

\subsection{Empirical Objects and Data Sources}

In this paper, 21 cities in Guangdong Province, China, are selected as the comparative subjects to carry out an empirical study on the cooperative relationship between regional STI platform and strategic emerging industries. As the Pioneer Area of China's reform and opening up, Guangdong's strategic emerging industries have good foundation, and the construction of regional STI platform and its supporting facilities are adequate. Its development is typical and forwardlooking for the whole country. At the same time, the regional economic development in Guangdong province is not balanced, the development of regional STI platform around the city construction and strategic emerging industries are in different stages of development and level, also has a strong reference to the research on the development and problems of China's strategic emerging industries to solve. The data of this study comes mainly from two aspects: part of data from the Chinese "Guangdong province science and Technology Statistics Yearbook" and the statistical yearbook of Guangdong province; the other part is derived from Guangdong Province Statistical Bureau official website and related science and technology management department official website.

\subsection{Descriptive Statistical Analysis}

About the development status of Guangdong science and STI platform and strategic emerging industries, based on related research, this paper carries out data collection and statistical analysis from two aspects of input and output, and the results are shown in Table 1. Specifically, based on the STI platform with the main function of scientific research, technological development and personnel training [3], the scientific research funding, research project investment, investment, talent team construction and research equipment and assets invested in a number of indicators to measure STI platform in financial, human and material resources in three aspects of innovation resources input [17]. It can be seen from the measurement results that the investment of scientific and technological 
Table 1. The descriptive statistics of the scientific and technological innovation platform in Guangdong and the development of strategic emerging industries.

\begin{tabular}{|c|c|c|c|c|}
\hline Target & Measure index & Mean value & Variance & Median \\
\hline \multirow{8}{*}{$\begin{array}{l}\text { Construction of STI } \\
\text { platform }\end{array}$} & Investment in scientific research (billion) & 37.30 & 8626.92 & 2.71 \\
\hline & Investment in scientific research projects (thousands of items) & 0.55 & 1.52 & 0.07 \\
\hline & Investment in talent and team construction (thousands of people) & 5.56 & 156.04 & 0.77 \\
\hline & R\&D equipment and asset input (billion) & 13.84 & 957.55 & 2.29 \\
\hline & Number of patent applications (thousands of items) & 2.94 & 36.65 & 0.17 \\
\hline & Financial efficiency of new product and new process (billion) & 376.04 & $770,046.97$ & 47.64 \\
\hline & Achievement Award (item) & 26 & 2185.96 & 5 \\
\hline & Training and providing industry talent output (thousands of people) & 6.50 & 303.96 & 0.21 \\
\hline \multirow{2}{*}{$\begin{array}{l}\text { The growth of strategic } \\
\text { emerging industries }\end{array}$} & Total industrial output value (billion) & 625.00 & $915,266.95$ & 3991.99 \\
\hline & Industrial added value (billion yuan) & 143.53 & $51,131.17$ & 937.72 \\
\hline
\end{tabular}

funds in the province is up to 3 billion 730 million RMB on average. The average investment in R\&D equipment and assets was up to 1 billion 384 million RMB. However, the variance is relatively large, which indicates that the innovation resources of the STI platform have a higher average level of investment, but the input of the inter regional platform is relatively unbalanced. In terms of output of STI platform, we can characterize and measure the innovation output performance of STI platform through multiple indicators such as patents [17], achievement award [18], achievement transformation [19], and training and providing industry talents [3]. The statistical results show that: The average level of innovation output of the STI platform of the whole province is higher, The average output of the new product and new technology has reached 37 billion 604 million RMB. At the same time, the output level of inter regional STI platform is relatively unbalance. Secondly, the growth of strategic emerging industries uses industrial output value to measure the industrial scale, and uses industrial added value to represent the results of industrial activities [20]. The statistical results show that the average level of new industries and activities in the province is relatively high, and the average industrial output value of the province is up to 62 billion 500 million RMB. The high variance indicates that the development of emerging industries in the region is unbalanced. On the whole, the STI platform and the development level of the strategic emerging industries are relatively high, but the regional development is not equal.

\subsection{Calculation Results and Discussion}

\subsubsection{The Comprehensive Level of Regional STI Platform and Strategic Emerging Industries Development}

Considering the comparability of data, this paper first standardized the original index data. At the same time, the initial normalized data value is added to 0.0001 to avoid the appearance of the meaningless situation in the calculation process. 
The original data are converted into dimensionless index evaluation values, That is, the values of each index are at the same number of levels. In addition, in order to avoid the deviation caused by a certain degree of subjective factors, the entropy weight method is used to determine the weight [21]. The comprehensive index of the construction of STI platform and strategic emerging industry growth were calculated by means of standardized data, the calculation results are shown in Table 2.

\subsubsection{The Coordination of STI Platform Construction and Strategic Emerging Industries Development}

Based on the relative development of the STI platform and the strategic emerging industries, we divide the types of co-coupling development. The comprehensive score and the comprehensive score of the platform represent the relative development level of the two subsystems in the whole system. The higher the score is, the higher the relative development level is. For this reason, this paper divides the development type of synergistic coupling development between regional STI platform and strategic emerging industry into "platform driven, industrial spillover, synchronous development and benign interaction". Accordingly, regional distribution is shown in Figure 1. Among them, 13 cities in the province belong to industrial spillover cities. The platform is far behind the industrial development, and the width of the synergistic coupling between regions is different. The middle and high level three are all distributed, while the regional synergistic coupling intensity is not very different as a whole, but most of the cities are generally low, mainly distributed between 0 and 0.3 . In addition, the development of innovation platform in Yunfu and Chaozhou is ahead of the development of new industries, which is a platform driven type, with the same

Table 2. Guangdong STI platform construction comprehensive index and strategic emerging industry growth comprehensive index.

\begin{tabular}{cccccc}
\hline $\begin{array}{c}\text { Region } \\
\text { (city) }\end{array}$ & $\begin{array}{c}\text { Comprehensive index of } \\
\text { the construction of STI } \\
\text { platform }\left(\mathrm{U}_{1}\right)\end{array}$ & $\begin{array}{c}\text { Comprehensive index } \\
\text { of strategic emerging } \\
\text { industry growth }\left(\mathrm{U}_{2}\right)\end{array}$ & Region & $\begin{array}{c}\text { Comprehensive index of } \\
\text { the construction of STI } \\
\text { platform }\left(\mathrm{U}_{1}\right)\end{array}$ & $\begin{array}{c}\text { Comprehensive index of } \\
\text { strategic emerging } \\
\text { industry growth }\left(\mathrm{U}_{2}\right)\end{array}$ \\
\hline Shenzhen & 0.800709 & 1.0001 & Shanwei & 0.006529 & 0.013043 \\
Guangzhou & 0.930179 & 0.561779 & Yunfu & 0.03644 & 0.004516 \\
Huizhou & 0.229938 & 0.225215 & Shaoguan & 0.007761 & 0.008123 \\
Foshan & 0.167695 & 0.349304 & Shantou & 0.002591 & 0.025085 \\
Zhongshan & 0.061032 & 0.195169 & Chaozhou & 0.00786 & 0.000512 \\
Zhuhai & 0.068739 & 0.11778 & Yangiang & 0.0001 & 0.017694 \\
Jieyang & 0.043772 & 0.064647 & Maoming & 0.0001 & 0.012468 \\
Jiangmen & 0.03293 & 0.071682 & Heyuan & 0.0001 & 0.011173 \\
Dongguan & 0.017602 & 0.259189 & Meizhou & 0.0001 & 0.001436 \\
Zhaoqing & 0.018324 & 0.068509 & Meizhou & 0.0001 & 0.000597 \\
Qingyuan & 0.009614 & 0.064186 & Guangdong Province & 0.1163 & 0.146295 \\
\hline
\end{tabular}




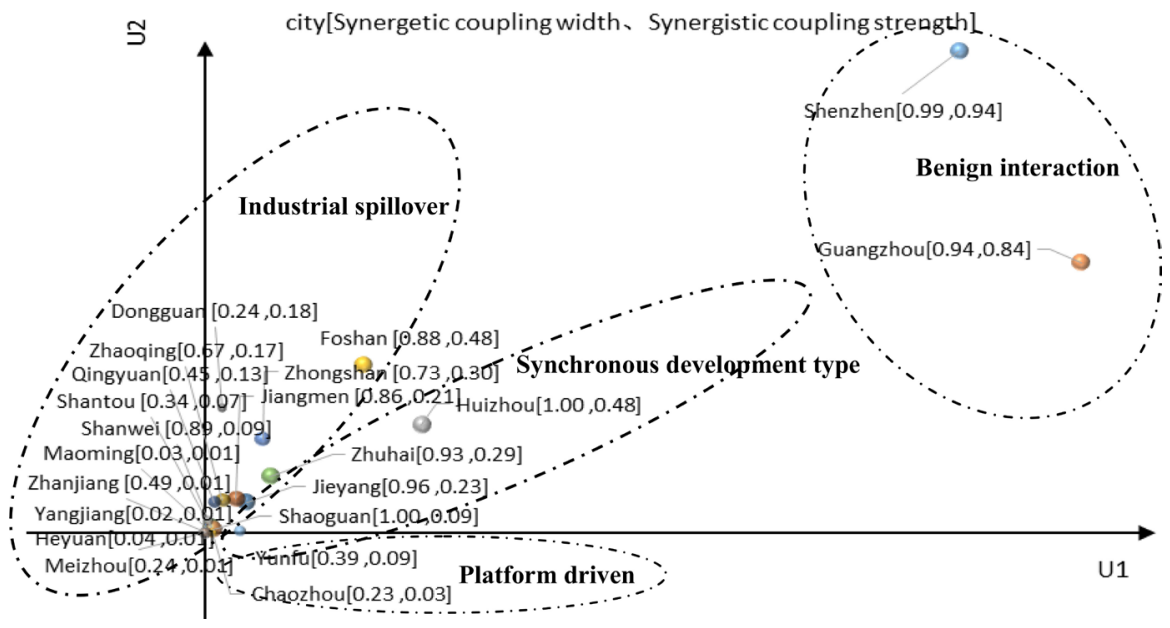

Figure 1. The Synergetic coupling development type of regional STI platform and strategic emerging industries in Guangdong Province.

width and intensity level, but all of them show a low level. Huizhou, Zhuhai and other cities are of synchronous development, showing a high-width, low-intensity development model. In addition to the above areas, Shenzhen and Guangzhou belong to the benign interaction type, and their synergistic coupling width and intensity level are relatively ahead of other cities in the province. The reason is that the allocation and layout of regional science and technology resources are not balanced and inadequate, so further optimization is needed in the future.

\subsubsection{Regional Collaborative Coupling Development Type Division and Difference}

Based on the coupling evaluation model, we calculated the width and intensity of synergistic coupling between STI platform and strategic emerging industry in 21 cities of Guangdong province (Table 3). As a whole, the synergistic coupling width and intensity of the STI platform and strategic emerging industries are low in Guangdong province. The area with the highest synergistic coupling width is Huizhou, followed by Shaoguan, Shenzhen, Jieyang, Guangzhou and so on, and the value is above 0.8 . Zhongshan and Zhaoqing are in a moderate level, between 0.6 and 0.8 . In contrast, the co-coupling width of other cities is relatively low, between 0 and 0.6 , and most of the regions are relatively backward. On the other hand, the area with the highest synergistic coupling strength is Shenzhen, followed by Guangzhou, which is 0.943062 and 0.836956 respectively, and at a high level. Huizhou and Foshan are in the middle level, and the value is between 0.4 and 0.6 . The other cities in the whole province were shown to be low level and distributed between 0 and 0.4 . This phenomenon was basically consistent with the current level of economic development in various regions. The result of cooperative coupling development is generally low, which indicates that the benign interaction mechanism of STI platform and strategic emerging industries has not yet formed in Guangdong provincial. The depth and level of interrelated and coordination between platforms and emerging industries are still low. 
Table 3. Synergistic coupling relationship results between regional STI platform and strategic emerging industry in Guangdong Province.

\begin{tabular}{cccccc}
\hline Region & $\begin{array}{c}\text { Synergetic } \\
\text { coupling width } \\
(\mathrm{C})\end{array}$ & $\begin{array}{c}\text { Synergistic } \\
\text { coupling } \\
\text { strength (D) }\end{array}$ & Region & $\begin{array}{c}\text { Synergetic } \\
\text { coupling } \\
\text { width (C) }\end{array}$ & $\begin{array}{c}\text { Synergistic } \\
\text { coupling } \\
\text { strength (D) }\end{array}$ \\
\hline Shenzhen & 0.98774 & 0.943062 & Shanwei & 0.889244 & 0.093287 \\
Guangzhou & 0.939029 & 0.836956 & Yunfu & 0.392402 & 0.089641 \\
Huizhou & 0.999892 & 0.477024 & Shaoguan & 0.99948 & 0.089093 \\
Foshan & 0.876606 & 0.476028 & Shantou & 0.339431 & 0.068535 \\
Zhongshan & 0.725883 & 0.304936 & Chaozhou & 0.229782 & 0.031015 \\
Zhuhai & 0.930868 & 0.294639 & Yangjiang & 0.022354 & 0.014102 \\
Jieyang & 0.962931 & 0.228473 & Maoming & 0.031574 & 0.014086 \\
Jiangmen & 0.862779 & 0.212435 & Heyuan & 0.03517 & 0.014079 \\
Dongguan & 0.2382 & 0.181565 & Meizhou & 0.243464 & 0.013674 \\
Zhaoqing & 0.665968 & 0.17004 & Meizhou & 0.491819 & 0.013088 \\
Qingyuan & 0.453209 & 0.129319 & Guangdong & 0.586563 & 0.223575 \\
\hline
\end{tabular}

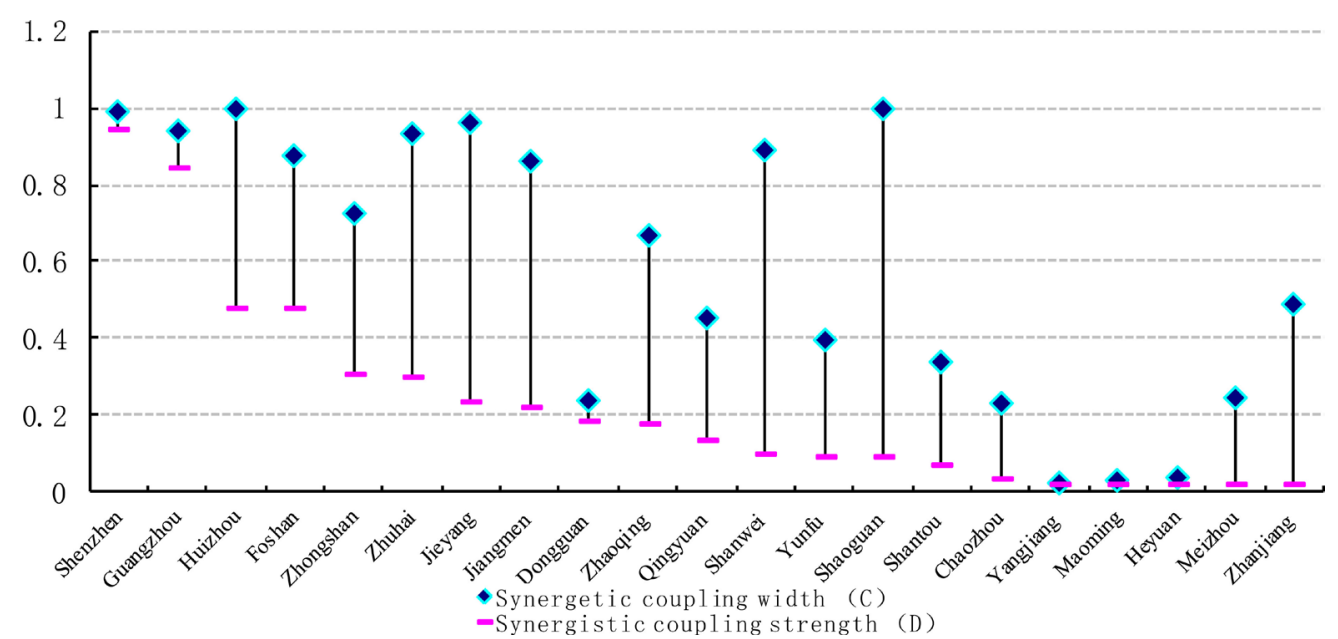

Figure 2. A synergistic coupling trend of the regional STI platform and strategic emerging industries in Guangdong Province.

Further, there is a great difference in the synergistic coupling development of Guangdong regional STI platform and strategic emerging industries, showing the current level of synergistic coupling strength lagging behind the level of synergistic coupling as shown in Figure 2. In the 21 cities surveyed, only two cities in Guangzhou and Shenzhen had the same width and intensity, and they were all at a high level, and the values ranged from 0.8 to 1 . The synergistic coupling width and the high level of Huizhou and Foshan, the intensity is relatively low, showing moderate level; while the synergistic coupling width in Zhuhai and Jieyang is at a high level, but the intensity is different from that of it, showing a low level. The coupling synergy width of other cities is basically distributed 
below the moderate level, mainly between 0 - 0.6. The synergistic coupling strength of these cities is below the width and basically at a low level, mainly between 0 and 0.2. So local governments should attach importance to the interaction and synergistic relationship between the construction of regional STI platform and strategic emerging industries. According to the local conditions, the corresponding cooperative coupling development strategy is made to realize the high synergy coupling of the construction of regional STI platform and strategic emerging industries as soon as possible.

\section{Conclusions and Recommendations}

Based on the analysis of the interaction relationship between regional STI platform and strategic emerging industries, this paper demonstrates the existence of interaction coupling between the two. Therefore, a collaborative coupling relationship measurement model of regional STI platform and strategic emerging industries is built. And the synergistic coupling strength of the 21 cities' STI platform and the strategic emerging industries was evaluated in Guangdong in 2015. The results show that: 1 ) on the whole, the synergistic coupling width and coordination strength of Guangdong's STI platform and strategic emerging industries are all low, and the two have not yet formed a benign interaction mechanism. 2) The matching differences of innovation platforms and industries in Guangdong province are quite different. 3) According to the relative relation between the development of the STI platform and the development of strategic emerging industries, Guangdong province regional STI platform and strategic emerging industries collaborative coupling development can be divided into four types: the first is the industry spillover type, the second is the simultaneous development, the third is the platform driven, and the fourth is the interaction of four types.

Therefore, in order to achieve mutual promotion and coordinated development of regional STI platform and strategic emerging industries, it depends on the effective cultivation and promotion of the government. For the industrial spillover, on the one hand, we should promote the industrial development in the aspects of fund support, technological innovation, intellectual property protection and talent training. At the same time, we should pay more attention to the positive guidance of the transformation of industrial scientific and technological achievements and the policy of industrialization, and strengthen the spillover effect of the industry to the platform. On the other hand, the layout of the platform is optimized and special funds are set up to support the platform to promote the development of the platform. For the cities of platform driven, we should support the development of innovative platforms by incentives or undertaking research projects and funds. We should support the platform bringing in high-level personnel and encourage social forces to participate in its construction, which can enhance the platform's capacity for continuous innovation and provide technology and industrial information services for industrial development. 
For the synchronous development city, the government should adhere to the synchronization of the development of the platform and the industry, adhere to the combination of unified planning and classification guidance, and pay attention to the guidance of the system. For the benign interactive city, on the one hand, the government should change its function properly to integrate the innovation platform of service and carry out the market operation of the platform. At the same time, the platform is introduced into the team of universities and research and development institutions to realize the sharing mechanism of the research results by the platform and the enterprise. On the other hand, we should pay attention to and guide the spillover effect of the platform and industry, and realize the transfer of knowledge and technology to the surrounding areas. On the other hand, we should pay attention to and guide the spillover effect of the platform and industry, and realize the transfer of knowledge and technology to the surrounding areas.

\section{References}

[1] Wang, L.F. and Fu, Y.L. (2013) Research on the Allocation of Scientific and Technological Resources in the Development of High-Tech Industries. Scientific Research Management, 2, 152-160.

[2] He, Y.T. and Yuan, W. (2010) The Relationship between the Construction of Technological Innovation Service Platform and the Cultivation and Development of Strategic Emerging Industries. China University Science and Technology and Industrialization, 7, 64-66.

[3] Li, Z., Wang, X. and Mou, R. (2012) Research on the Construction of Technology Innovation Service Platform for China's Key Industries. Science and Technology Management, No. 3, 33-38.

[4] Nelson, R.R. (1995) Recent Evolutionary Theorizing about Economic Change. Journal of Economic Literature, 18, 48-90.

[5] Yue, Z.G. (2014) Research on the Collaborative Development of Technology Chain and Industrial Chain in Strategic Emerging Industries. Science and Technology Management, 2, 154-161.

[6] Huang, R.C. and Yang, X.J. (2014) Analysis on the Co-Evolution of Emerging Technologies and Emerging Industries. Technological Progress and Countermeasures, 3, 72-78.

[7] Wang, X.T. and Hu, S.H. (2007) Construction of an Industrial Technology Innovation Platform for Independent Development. Science and Technology Management, 2, 103-106.

[8] Zheng, X.B. and Lu, L.J. (2011) Research on Regional Innovation Platform under the Perspective of Industrial Cluster Transformation and Upgrading. Science and Technology Management, 8, 65-70, 106.

[9] Wang, S.Y., Huo, G.Q., Sun, H. and Yang, Y. (2014) Research on the Life Cycle and Evolution of Strategic Emerging Industries-A Case Study Based on the Backtracking of British and American Leading Industries. Scientific Research, 11, 1630-1638.

[10] Wang, Y.C. and Wang, Q.Z. (1999) A New Definition of Coupling Degree and Its Application. Journal of Huaqiao University (Natural Science Edition), 3, 59-63. 
[11] Song, D. (2015) Coupling Effect of Technological Innovation and Strategic Emerging Industries. Technical Economics and Management Research, No. 5, 121-124.

[12] Lu, Y. and Di, Z. (2013) Research on the Coupling Development of Regional Technological Innovation and Strategic Emerging Industries. Industrial Technology Economy, 10, 90-100.

[13] Wu, D., Cao, L. and Chen, L. (1990) Principles and Application of Collaborative Learning. Huazhong University of Science and Technology press, Wuhan.

[14] Liu, N., Wang, X. and Han, H. (2015) Research on the Spatial and Spatial Characteristics and Driving Mechanism of the Collaborative Coupling between Science and Technology Innovation and High-Tech Industry Innovation. Science and Technology Management, 10, 59-70.

[15] Fan, B., Zhang, W. and He, J. (2013) Research on the Connotation and Measurement of the Coordinated Development of Economic and Social Development in China. Statistical Research, No. 7, 3-8.

[16] Hao, S., Yu, B. and Wu, W. (2009) Evaluation of the Coupling Degree of Enterprise Network Capability and Technical Ability. Scientific Research, No. 2, 250-254.

[17] Wang, L. and Liu, J. (2010) Construction and Application of Performance Evaluation Index System of University Science and Technology Innovation Platform. Science and Technology Management, No. 2, 110-112 + 123.

[18] Su, Y. (2013) Research on Theoretical Analysis of Collaborative Innovation Based on System Science. Scientific Research Management, 34, 140-144.

[19] Wang, B. and Tan, Q. (2013) Evaluation Index System of Industrial Innovation Platform and Its Weight Setting Research. Science and Technology Management, 12, 63-68.

[20] Lu, W., guan, X. and huang, R. (2013) Coordinated Development of Technological Innovation and Strategic Emerging Industries. Technical Economy, 7, 13-17 + 89.

[21] Ren, S. (2014) Research on Regional Differences in Technological Innovation Capability of Enterprises Based on Entropy Method. Statistics and Decision, 16, 178-181. 